\title{
The effect of lower legs intermittent sequential pneumatic compression (ISPC)
device on FloTrac: report of two cases in living donor liver transplantation \\ The effect of lower legs intermittent sequential pneumatic compression (ISPC)
device on FloTrac: report of two cases in living donor liver transplantation
}

Hao-Hu Chen ${ }^{1}$, I-Cheng Lu², Kuang-I Cheng 1,2

Department of Anesthesiology, Kaohsiung Medical University Hospital, Kaohsiung Medical University, Kaohsiung, Taiwan

2 Faculty of Anesthesiology, College of Medicine, Kaohsiung Medical University, Kaohsiung, Taiwan

\section{Background:}

Hemodynamic monitoring and venous thromboembolism prophylaxis are important issues in liver transplantation (LT). In our hospital, intermittent sequential pneumatic compression (ISPC) device over lower legs (Fig. 1, Fig. 2) and FloTrac (Edwards Lifesciences, version 4.0) are applied simultaneously in LT. Herein we present the effect of ISPC devices assessed by FloTrac during LT.

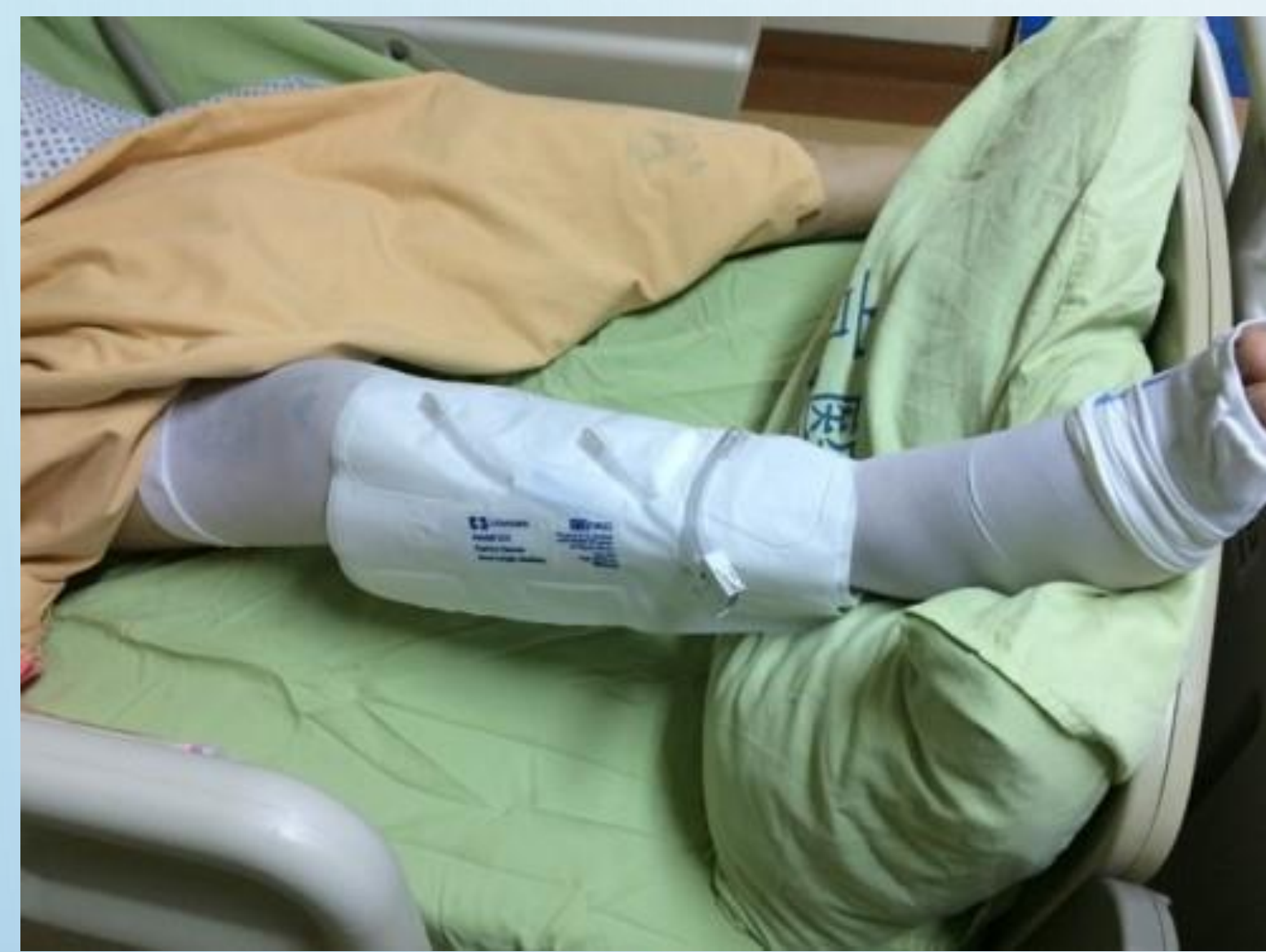

Figure 1. compression stockings and ISPC sleeve (Kendall SCD Express Sleeves, Knee length, Medium, Recorder REF 73022)

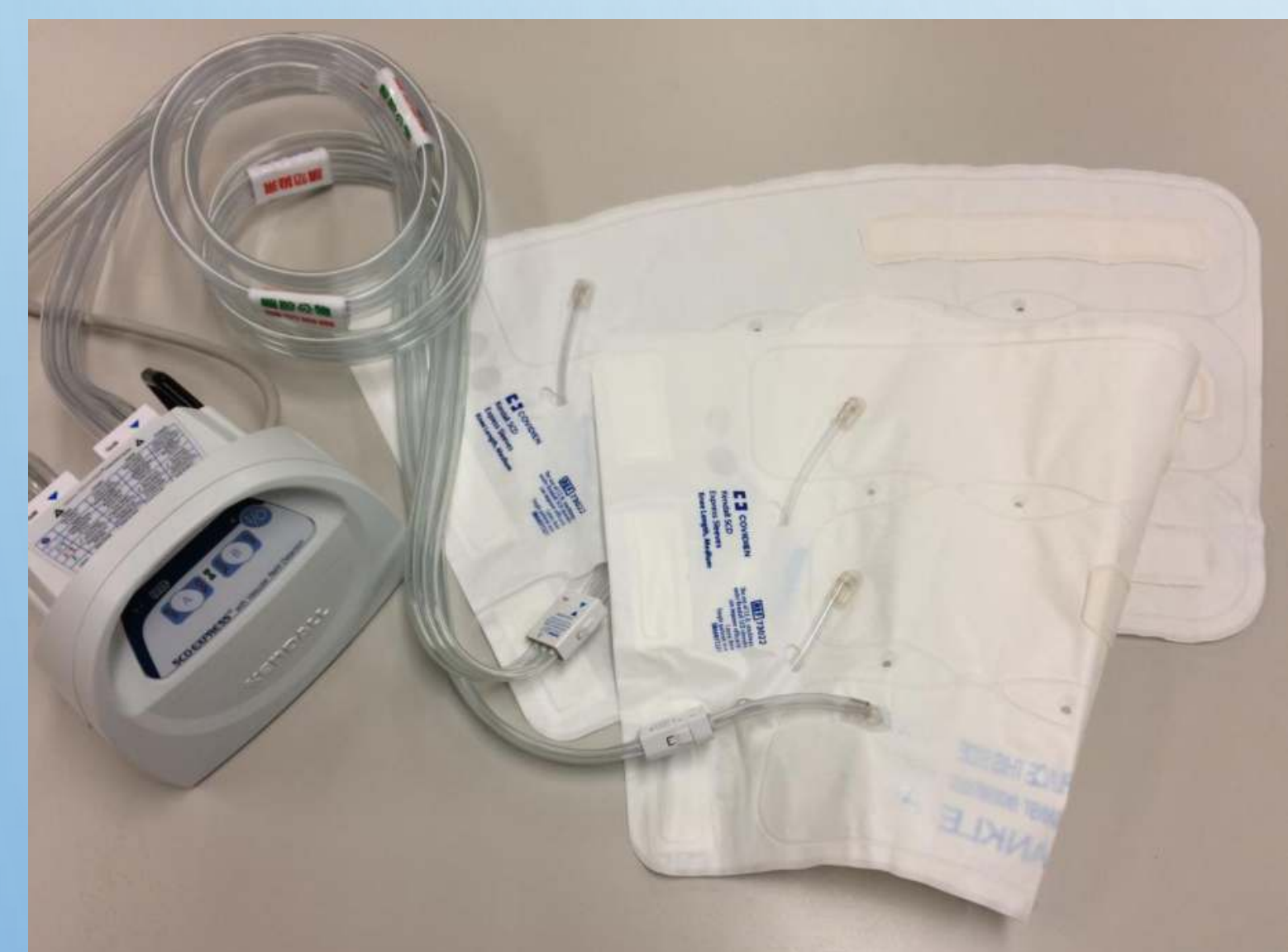

Figure 2. ISPC sleeve and Kendall SCD Express Compression system (Covidien Ltd, Mansfield, MA, USA)

\section{Case Report:}

The donor was a 20-year-old male. The recipient was a 60year-old male with history of hepatocellular carcinoma, hepatitis C, liver cirrhosis Child-Pugh A, and the MELD score was 11 . The surgery took 9 hours in donor and 12 hours in recipient. The blood loss was $575 \mathrm{ml}$ and $1910 \mathrm{ml}$, respectively. Both patients wore graduated compression stockings and ISPC sleeves over lower legs (Fig. 1). The ISPC sleeves were operated by Kendall SCD Express Compression system (Fig. 2). FloTrac was used for hemodynamic monitoring.

After ISPC was activated, heart rate (HR), mean arterial pressure (MAP), cardiac index $(\mathrm{Cl})$, stroke volume variation (SVV), systemic vascular index (SVRI) remained unchanged in donor (Fig. 3). In recipient, HR and SVRI remained unchanged, but SVV elevated, MAP and Cl decreased (Fig. 4).

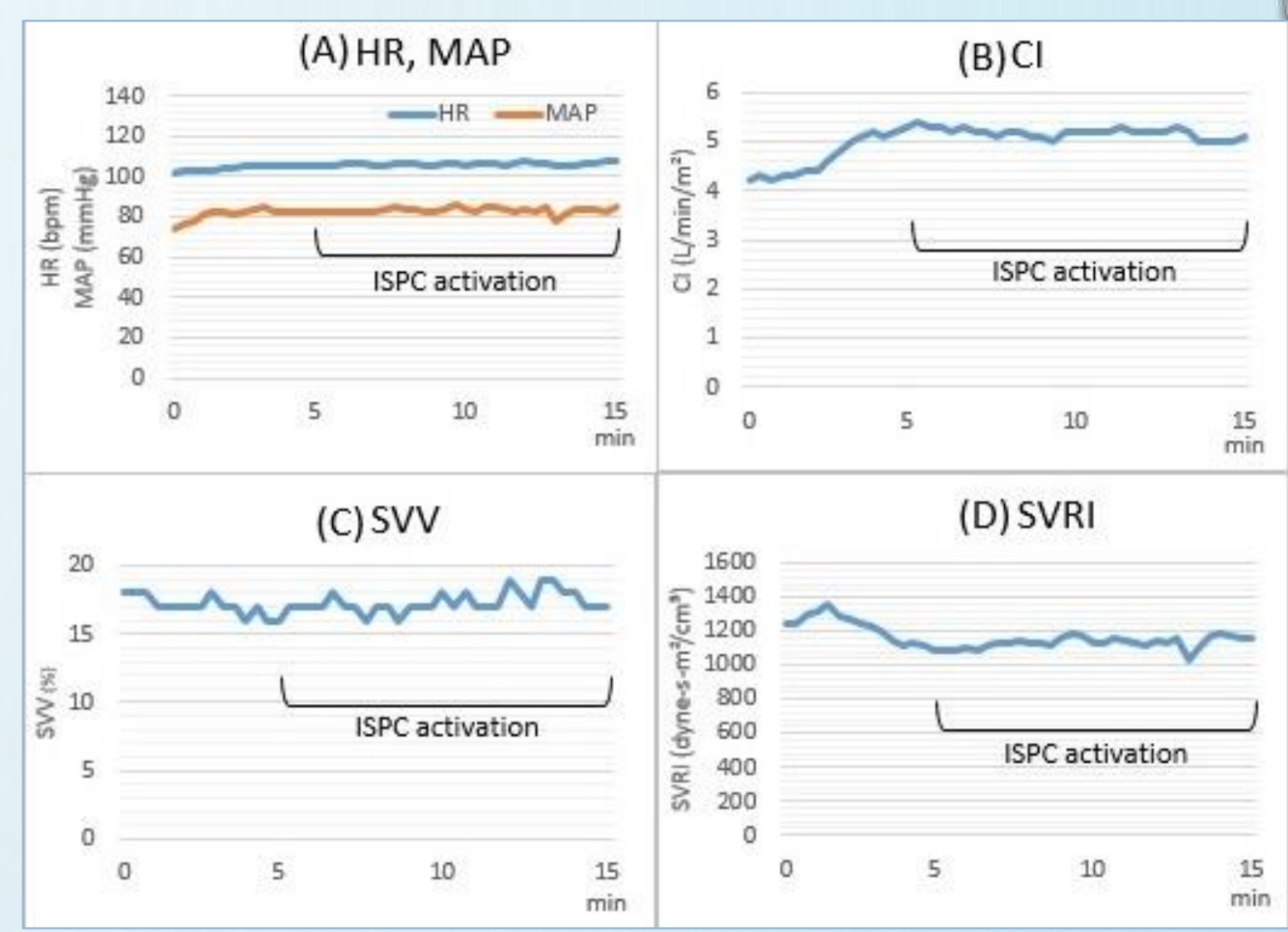

Figure 3. Donor's hemodynamic change. (A) Heart rate (HR), mean arterial pressure (MAP), (B) cardiac index (CI), (C) stroke volume variation (SVV), (D) systemic vascular resistance index (SVRI).



(C) SVV



(B) $\mathrm{Cl}$



(D) SVRI

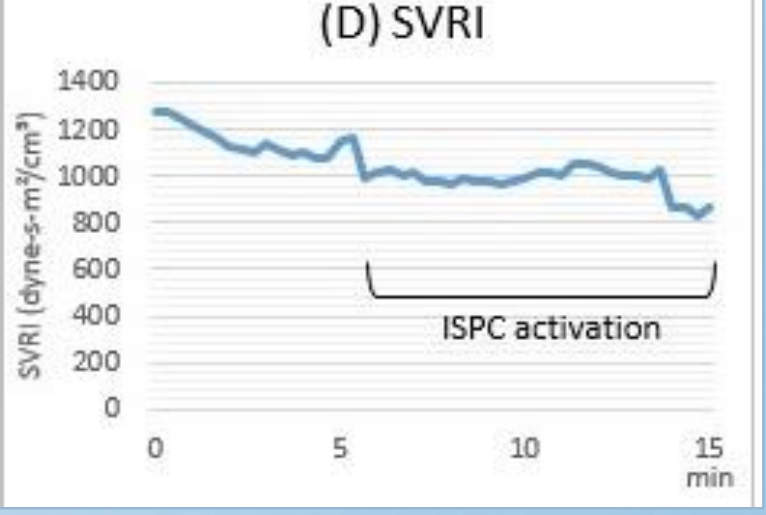

Figure 4. Recipient's hemodynamic change. (A) Heart rate (HR), mean arterial pressure (MAP), (B) cardiac index (CI), (C) stroke volume variation (SVV), (D) systemic vascular resistance index (SVRI).

\section{Discussion:}

Previous study showed the activation of ISPC augmented cardiac output ${ }^{1}$. However, the results in our cases were different. Possible reasons include: First, the volume of calf was small in comparison to that of the whole leg. The compression stockings reduced the calf volume and further attenuated the effect of ISPC. Second, healthy people were able to compensate the effect of ISPC. In conclusion, longer ISPC sleeves may be beneficial on hemodynamics for LT recipient. Further studies are required to assess how ISPC devices affect FloTrac parameters.

\section{References:}

1. BICKEL, Amitai, et al. The physiological impact of intermittent sequential pneumatic compression (ISPC) sleeves on cardiac activity. The American Journal of Surgery, 2011, 202.1: 16-22.



